\title{
Filter-based turbo equalization for TCM signals
}

\author{
Pei Xiao, Rolando Carrasco \\ School of Electrical, Electronic and Computer Engineering \\ University of Newcastle, NE1 7RU, United Kingdom \\ E-mail: pei.xiao, r.carrasco@ncl.ac.uk \\ Ian Wassell \\ Lab for Communication Engineering, University of Cambridge \\ 15 JJ Thomson Avenue, CB3 0FD, United Kingdom \\ E-mail: ijw24@eng.cam.ac.uk
}

\begin{abstract}
In this paper, we presents a novel method of turbo equalization and decoding multilevel trellis coded modulation (TCM) signals over frequency selective channels. Results show that the proposed algorithm achieves better performance with reduced complexity compared to previous work on the MMSE filter-based turbo equalization for non-binary coded modulation scheme. The performance gain is accomplished by passing the refined signal from different paths to the TCM decoder as channel value in addition to the a prior information. While the computational complexity is reduced by avoiding matrix inversion for each symbol estimate.
\end{abstract}

Keywords - turbo equalization, TCM, Log-MAP, interference cancellation.

\section{INTRODUCTION}

In a cellular mobile communications environment, multipath propagation causes dispersion of transmitted signals. The time delay spread causes intersymbol interference (ISI) and degrades system performance. Therefore, equalization methods which can mitigate the effects of ISI must be employed. A frequency selective channel can be described as a rate one convolutional code defined over the field of real or complex numbers, so the combination of a channel code and ISI channel can be viewed as a serial concatenation system, and can be decoded using turbo processing principle. Douillard, et al. proposed turbo equalization in [1] which combines equalization with channel decoding to remove the effect of intersymbol interference caused by frequency selective fading channels. The receiver consists of two trellisbased detectors, one for equalization and one for decoding. It is shown that turbo equalization significantly improve the performance over separate equalization and decoding. However, for channels with large delay spreads and for large constellation sizes, the trellis based turbo equalization scheme suffers from prohibitive computational complexity. In $[2,3]$, Wang, Tuchler, et al, introduced new approaches to combining equalization based on the linear MMSE filtering with decoding. It was shown that this new approach provides a tremendous complexity reduction with acceptable performance degradation compared to the trellis-based approach.

TCM is a powerful technique to improve bandwidth efficiency without increasing the transmitted power. It com- bines modulation and coding by optimizing the Euclidean distance between codewords. Turbo equalization was applied to TCM in several papers, e.g., [4,5]. Trellis-based equalization was used in the above mentioned papers and references therein. Despite of its good performance, the major disadvantage is the exponentially increasing complexity of the equalizer for channels with long impulse response or large signal constellation size. To reduce the complexity, the MMSE filter-based turbo equalization originally proposed in $[2,3]$ was extended to the TCM signals in $[6,7]$. Although it reduces the complexity from exponential to $\mathrm{cu}-$ bic compared to the trellis-based approach, this algorithm is still computationally intensive due to matrix inversion at each time instant.

This paper aims at further reducing the complexity of turbo equalization for TCM signals. This is accomplished by obtaining symbol a posteriori probabilities directly based on the output of the interference canceller, thus the MMSE filtering and its inherent matrix inversion procedure is avoided. In order to further improve the system performance, we modify the TCM decoder such that it takes the refined channel value in addition to symbol a posteriori probabilities as inputs and outputs both symbol extrinsic probabilities and log-likelihood ratio (LLR) values for information bits. This is in contrast to previous work on filter-based approach in which the TCM decoder only takes symbol a posteriori probabilities as input. A comparison between the proposed scheme and the trellis-based as well as other existing filter-based equalization schemes is provided in this paper to demonstrate the efficiency of the proposed algorithm.

The transmission system under study is shown in Figure 1. The information sequence is TCM encoded and mapped into one of the PSK or QAM signals. For simplicity, we consider Ungerboeck's 4-state, 8-PSK scheme [8]. In such a case, each pair of information bits $b_{n}^{0}, b_{n}^{1}$ at time instant $n$ correspond to one of $M=8$ PSK symbols, denoted by $s_{n}$. The coded and modulated symbol sequence $\left\{s_{n}\right\}$ is block interleaved and transmitted over the ISI channel, which can be modeled by an equivalent baseband system where the transmit filter, the channel and the receive filter, are represented by a discrete-time $(L+1)$-tap transversal filter with finite-length impulse response $h_{n}=\sum_{l=0}^{L} h_{l} \delta_{n-l}$ where the complex channel coefficients $h_{l}$ are assumed time invariant. The channel output sequence $\left\{r_{n}\right\}$ can be ex- 


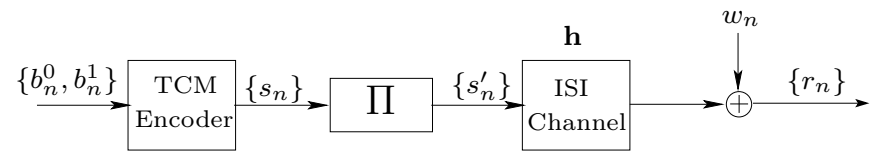

Fig. 1. Block diagram of transmitter.

pressed as

$$
r_{n}=\sum_{l=0}^{L} s_{n-l}^{\prime} h_{l}+w_{n}
$$

where $s_{n-l}^{\prime}$ is the coded and interleaved symbol, and $w_{n}$ is complex additive white Gaussian noise with zero mean and variance $N_{0}$.

\section{Filter Based Equalization And TCM DECODING}

The proposed equalization and TCM decoding algorithm is depicted in Figure 2. In the first stage of the turbo equalization, we use a simple decision feedback equalizer (DFE) to obtain an initial estimate of the transmitted symbols. The equalizer coefficients are obtained adaptively using recursive least square (RLS) algorithm. The channel is also estimated using pilot symbols at this stage. With symbol and channel estimates, we perform interference cancellation and pass the refined (combined) signal to the TCM decoder. In the meantime, the a posteriori symbol probabilities at each time instant $n$ are collected in an M-ary vector and passed to the decoder as a priori information. The TCM decoder computes the extrinsic probability of the TCM symbols as well as the LLR of the information bits. The equalization and decoding blocks will be described in details next.

\section{A. Equalization Algorithm}

In order to reduce the computational complexity, we replace the trellis decoding with a soft interference canceller (SIC) followed by i) a Log-MAP mapper to compute a posteriori probabilities; ii) a maximum ratio combiner (MRC) to obtain a refined channel value for the TCM decoder based on the interference cancelled signal. This algorithm is different from the ones presented in $[2,3]$, since modifications have to made here to accommodate multilevel modulation. In particular, one has to compute extrinsic probabilities rather than log-likelihood ratios (LLRs) in order to allow the application of turbo equalization to TCM systems; while methods based on the computation of LLRs assume a binary alphabet of data.

Based on (1), the ISI cancelled signals after performing interference cancellation using soft estimates of the transmitted symbols become

$$
\begin{aligned}
\tilde{r}_{n} & =h_{0} s_{n}^{\prime}+\left(h_{1} s_{n-1}^{\prime}-\hat{h}_{1} \bar{s}_{n-1}^{\prime}\right)+\ldots+w_{n} \\
\tilde{r}_{n+1} & =h_{1} s_{n}^{\prime}+\left(h_{0} s_{n+1}^{\prime}-\hat{h}_{0} \bar{s}_{n+1}^{\prime}\right)+\ldots+w_{n+1} \\
\ldots & \\
\tilde{r}_{n+L} & =h_{L} s_{n}^{\prime}+\left(h_{0} s_{n+L}^{\prime}-\hat{h}_{0} \bar{s}_{n+L}^{\prime}\right)+\ldots+w_{n+L}
\end{aligned}
$$

where $\hat{h}_{l}$ is the estimate of the channel coefficient $h_{l}$ and $\bar{s}_{n+i}^{\prime}$ denotes the soft estimate of $s_{n+i}^{\prime}$, which is computed as

$$
\bar{s}_{n+i}^{\prime}=\sum_{q=1}^{M} P\left(s_{n+i}^{\prime}=s_{q}\right) s_{q}
$$

The above formulas can be written in vector form as

$$
\tilde{\mathbf{r}}_{n}=\left[\begin{array}{c}
\tilde{r}_{n} \\
\tilde{r}_{n+1} \\
\vdots \\
\tilde{r}_{n+L}
\end{array}\right]=\left[\begin{array}{c}
h_{0} \\
h_{1} \\
\vdots \\
h_{L}
\end{array}\right] s_{n}^{\prime}+\left[\begin{array}{c}
v_{n} \\
v_{n+1} \\
\vdots \\
v_{n+L}
\end{array}\right]=\mathbf{h} s_{n}^{\prime}+\mathbf{v}
$$

where $\mathbf{h}=\left[h_{0}, \ldots, h_{L}\right]^{T}$ denotes the channel vector, and $\mathbf{v}=\left[v_{n}, \ldots, v_{n+L}\right]^{T}$ stands for the vector of combined noise and interference cancellation residual. This interference cancellation procedure is similar to the one proposed in $[6,7]$. Next, we shall introduce a different approach to compute the symbol a posteriori probabilities without using a MMSE filter.

Approximate each element of $\mathbf{v}$ as zero mean Gaussian random variable with variance $N_{v}$, the conditional pdf of $\tilde{\mathbf{r}}$ can be represented by

$$
\begin{aligned}
& p\left(\tilde{\mathbf{r}}_{n} \mid s_{n}^{\prime}=s_{m}\right)=\frac{1}{\left(\pi N_{v}\right)^{L+1}} \exp \left(-\frac{\left\|\tilde{\mathbf{r}}_{n}-\mathbf{h} s_{m}\right\|^{2}}{N_{v}}\right) \\
& =\frac{1}{\left(\pi N_{v}\right)^{L+1}} \exp \left(-\frac{\left\|\tilde{\mathbf{r}}_{n}\right\|^{2}+\left\|\mathbf{h} s_{m}\right\|^{2}-2 \operatorname{Re}\left\{\left(\mathbf{h} s_{m}\right)^{*} \tilde{\mathbf{r}}_{n}\right\}}{N_{v}}\right)
\end{aligned}
$$

where the superscript operator ()$^{*}$ is the conjugate transpose operation when applied to matrices, and simply the conjugate when applied to scalars. Note that

$$
\begin{aligned}
& P\left(s_{n}^{\prime}=s_{m} \mid \tilde{\mathbf{r}}_{n}\right)=\frac{p\left(\tilde{\mathbf{r}}_{n} \mid s_{n}^{\prime}=s_{m}\right) P\left(s_{n}^{\prime}=s_{m}\right)}{p\left(\tilde{\mathbf{r}}_{n}\right)} \\
& p\left(\tilde{\mathbf{r}}_{n}\right)=\sum_{q=1}^{M} P\left(s_{n}^{\prime}=s_{q}\right) p\left(\tilde{\mathbf{r}}_{n} \mid s_{n}^{\prime}=s_{q}\right)
\end{aligned}
$$

In a PSK constellation, all the signals lie on the unit circle, therefore have the same energy, i.e., $E_{s}=\left|s_{1}\right|^{2}=$ $\ldots=\left|s_{M}\right|^{2}$, and $\left\|\tilde{r}_{n}\right\|^{2}$ in (2) is the same for all the signal alternatives. Combining (2) and (3) yields

$P\left(s_{n}^{\prime}=s_{m} \mid \tilde{\mathbf{r}}_{n}\right)=\frac{P\left(s_{n}^{\prime}=s_{m}\right) \exp \left(2 \operatorname{Re}\left\{\left(\mathbf{h} s_{m}\right)^{*} \tilde{\mathbf{r}}_{n}\right\} / N_{v}\right)}{\sum_{q=1}^{M} P\left(s_{n}^{\prime}=s_{q}\right) \exp \left(2 \operatorname{Re}\left\{\left(\mathbf{h} s_{q}\right)^{*} \tilde{\mathbf{r}}_{n}\right\} / N_{v}\right)}$

Direct implementation of (4) leads to a numerically unstable algorithm. The problem can be tackled by performing the calculation in the log domain. With Log-MAP algorithm, each a posteriori probability is determined as

$P\left(s_{n}^{\prime}=s_{m} \mid \tilde{\mathbf{r}}_{n}\right)=$

$\frac{e^{\frac{2 \operatorname{Re}\left\{\left(\mathbf{h} s_{m}\right)^{*} \tilde{\mathbf{r}}_{n}\right\}}{N_{v}}+\log P\left[s_{n}^{\prime}=s_{m}\right]}}{e^{\max ^{*}\left(\frac{2 \operatorname{Re}\left\{\left(\mathbf{h} s_{1}\right)^{*} \tilde{\mathbf{r}}_{n}\right\}}{N_{v}}+\log P\left[s_{n}^{\prime}=s_{1}\right], \cdots, \frac{2 \operatorname{Re}\left\{\left(\mathbf{h} s_{M}\right)^{*} \tilde{\mathbf{r}}_{n}\right\}}{N_{v}}+\log P\left[s_{n}^{\prime}=s_{M}\right]\right)}}$ 


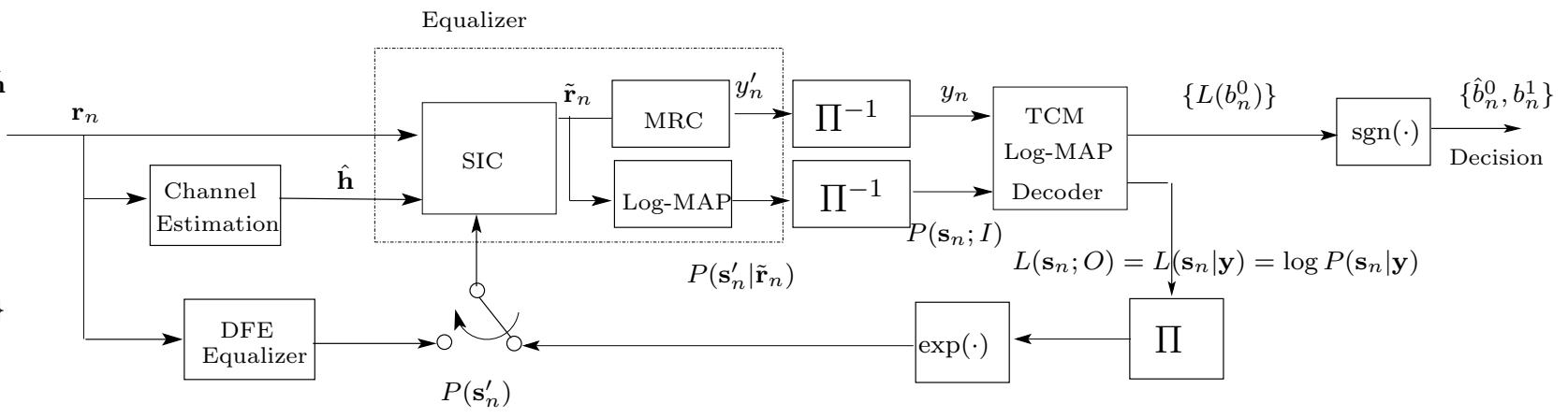

Fig. 2. Block diagram for the proposed turbo equalization scheme.

where the function $\max ^{*}()$ is defined as

$$
\max ^{*}(x, y)=\ln \left(e^{x}+e^{y}\right)=\max (x, y)+\ln \left(1+e^{-|x-y|}\right)
$$

which is max operation compensated with a correction term $\ln \left(1+e^{-|x-y|}\right)$. Also $\max ^{*}(x, y, z)=\max ^{*}\left[\max ^{*}(x, y), z\right]$. Note that symbols are assumed to be equally probable at the first iteration, i.e., $P\left(s_{n}^{\prime}=s_{1}\right)=P\left(s_{n}^{\prime}=s_{2}\right)=\ldots=$ $P\left(s_{n}^{\prime}=s_{M}\right)=1 / M$.

The vectors $P\left(\mathbf{s}_{n}^{\prime}\right)$ and $P\left(\mathbf{s}_{n}^{\prime} \mid \tilde{\mathbf{r}}_{n}\right)$ are defined as

$$
\begin{aligned}
P\left(\mathbf{s}_{n}^{\prime}\right) & =\left[\begin{array}{lll}
P\left(s_{n}^{\prime}=s_{1}\right) & \ldots & P\left(s_{n}^{\prime}=s_{M}\right)
\end{array}\right]^{T} \\
P\left(\mathbf{s}_{n}^{\prime} \mid \tilde{\mathbf{r}}_{n}\right) & =\left[\begin{array}{llll}
P\left(s_{n}^{\prime}=s_{1} \mid \tilde{\mathbf{r}}_{n}\right) & \ldots & P\left(s_{n}^{\prime}=s_{M} \mid \tilde{\mathbf{r}}_{n}\right)
\end{array}\right]^{T}
\end{aligned}
$$

The former is the a priori probabilities vector of the symbol $s_{n}^{\prime}$. It is an input to the SIC block. The latter is the output of the Log-MAP mapper followed by the SIC. Each element of $P\left(\mathbf{s}_{n}^{\prime}\right)$ is initialized as $P\left(s_{n}^{\prime}=s_{m}\right)=$ $\left\{\begin{array}{ll}1 & \text { if } \hat{s}_{n}^{\prime}=s_{m}, \\ 0 & \text { otherwise }\end{array}\right.$ where $\hat{s}_{n}^{\prime}$ is the hard decision from the DFE equalizer. At the following stages, this a priori probability vector is formed by the extrinsic information provided by the decoding step, i.e., $P\left(\mathbf{s}_{n}^{\prime}\right)=\Pi\left\{P\left(\mathbf{s}_{n} ; O\right)\right\}$, where $P\left(\mathbf{s}_{n} ; O\right)=\exp \left[L\left(\mathbf{s}_{n} ; O\right)\right]$ is the output of the TCM LogMAP decoder. We use the notations $P\left(\mathbf{s}_{n} ; I\right), P\left(\mathbf{s}_{n} ; O\right)$ to denote the input and output of the TCM decoder.

The refined signal to be passed to the TCM decoder as channel value is obtained based on the interference cancelled signal vector $\tilde{\mathbf{r}}_{n}$ using maximum ratio combining, i.e.,

$$
y_{n}^{\prime}=\hat{\mathbf{h}}^{*} \tilde{\mathbf{r}}_{n}=\hat{\mathbf{h}}^{*} \mathbf{h} s_{n}^{\prime}+\hat{\mathbf{h}}^{*} \mathbf{v}=\hat{\mathbf{h}}^{*} \mathbf{h} s_{n}^{\prime}+\eta^{\prime}
$$

where $\hat{\mathbf{h}}$ is an estimate of $\mathbf{h}, \eta^{\prime}$ is zero mean Gaussian random variable with variance $N_{\eta}=\mathcal{P} N_{0}$ and $\mathcal{P}=\sum_{l=0}^{L}\left|h_{l}\right|^{2}$ is the total received power from all the paths.

In contrast to the MMSE filter-based approach introduced in $[6,7]$, the derivation of symbol a posteriori probabilities described above does not involve matrix inversion. The presented algorithm reduces the computational complexity from $O\left(L^{3}\right)$ to $O\left(L^{2}\right)$, where $L$ is the number of channel taps.

\section{B. Decoding Algorithm}

The proposed TCM Log-MAP decoder takes the deinterleaved probability vector $P\left(\mathbf{s}_{n} ; I\right)=\Pi^{-1}\left\{P\left(\mathbf{s}_{n}^{\prime} \mid \tilde{\mathbf{r}}_{n}\right)\right\}$ as priori information as well as the combined signal $y_{n}=$ $\Pi^{-1}\left(y_{n}^{\prime}\right)$ as channel input. It outputs the log-likelihood ratio of the information bits $\left\{L\left(b_{n}^{0}\right), L\left(b_{n}^{1}\right)\right\}$ and the refined probability vector $P\left(\mathbf{s}_{n} ; O\right)=P\left(\mathbf{s}_{n} \mid \mathbf{y}\right)$ based on the code constraints and the trellis structure of the TCM code where $\mathbf{y}=\left[\begin{array}{llll}y_{1} & y_{2} & \ldots & y_{L_{b}}\end{array}\right]$ and $L_{b}$ denotes the symbol block length. Each element of $\mathbf{y}$ is defined as

$$
y_{n}=\Pi^{-1}\left(y_{n}^{\prime}\right)=\Pi^{-1}\left(\hat{\mathbf{h}}^{*} \mathbf{h} s_{n}^{\prime}+\eta^{\prime}\right)=\hat{\mathbf{h}}^{*} \mathbf{h} s_{n}+\eta
$$

The LLR values $\left\{L\left(b_{n}^{0}\right), L\left(b_{n}^{1}\right)\right\}$ are used for making hard decision on the transmitted information bits; while the logarithm of extrinsic probability $L\left(\mathbf{s}_{n} ; O\right)=L\left(\mathbf{s}_{n} \mid \mathbf{y}\right)=$ $\log P\left(\mathbf{s}_{n} \mid \mathbf{y}\right)$ is fed back to equalizer as a priori probability vector for the next iteration of turbo equalization and TCM decoding.

Let us denote $\alpha_{n}\left(\sigma^{\prime}\right)$ as the state metric at the $n^{\text {th }}$ trellis node, and $\beta_{n+1}(\sigma)$ as the state metric for the portion of the trellis beyond the $n^{t h}$ trellis node, where $\sigma^{\prime}$ and $\sigma$ are the generic states at the $n^{\text {th }}$ and $(n+1)^{\text {th }}$ nodes, respectively. The output $P\left(s_{n}=s_{m} \mid \mathbf{y}\right)$ is computed according to BCJR algorithm [9] (since two dimensional TCM constellation is employed, a non-binary version of BCJR is considered)

$$
\begin{aligned}
P\left(s_{n}\right. & \left.=s_{m} \mid \mathbf{y}\right)=\sum_{\sigma^{\prime}, \sigma: s_{m}} P\left(\sigma^{\prime}, \sigma \mid \mathbf{y}\right)=\frac{1}{P(\mathbf{y})} \sum_{\sigma^{\prime}, \sigma: s_{m}} P\left(\mathbf{y}, \sigma^{\prime}, \sigma\right) \\
& =\frac{\sum_{\sigma^{\prime}, \sigma: s_{m}} \alpha_{n}\left(\sigma^{\prime}\right) \gamma_{n}^{*}\left(\sigma^{\prime}, \sigma\right) \beta_{n+1}(\sigma)}{\sum_{\sigma^{\prime}, \sigma} \alpha_{n}\left(\sigma^{\prime}\right) \gamma_{n}\left(\sigma^{\prime}, \sigma\right) \beta_{n+1}(\sigma)}
\end{aligned}
$$

where $\gamma_{n}\left(\sigma^{\prime}, \sigma\right), \gamma_{n}^{*}\left(\sigma^{\prime}, \sigma\right)$ are the metrics for the branch connecting state $\sigma^{\prime}$ at node $n$ and $\sigma$ at node $(n+1)$. Their detailed expressions will be given later on. The notation $\sigma^{\prime}, \sigma: s_{m}$ denotes the sets of state pairs $\left(\sigma^{\prime}, \sigma\right)$ in the TCM trellis corresponding to symbol $s_{m}$, and $\sigma^{\prime}, \sigma$ denotes all the possible sets of state pairs where the transition $\left(\sigma^{\prime}, \sigma\right)$ is possible.

Now, we modify the TCM decoder in order to be able to get the soft outputs of information bits. Let $\mathcal{B}+$ be the set of state pairs $\left(\sigma^{\prime}, \sigma\right)$ such that the first info bit $b_{n}^{0}$ at time $n$ is +1 . Similarly define $\mathcal{B}^{-}$. The LLR value of the first info 
bit $b_{n}^{0}$ at the output of the TCM decoder is given by

$$
\lambda\left(b_{n}^{0}\right)=\log \frac{\sum_{\mathcal{B}^{+}} \alpha_{n}\left(\sigma^{\prime}\right) \gamma_{n}\left(\sigma^{\prime}, \sigma\right) \beta_{n+1}(\sigma)}{\sum_{\mathcal{B}^{-}} \alpha_{n}\left(\sigma^{\prime}\right) \gamma_{n}\left(\sigma^{\prime}, \sigma\right) \beta_{n+1}(\sigma)}
$$

The soft value of the second info bit $b_{n}^{1}$ corresponding to the TCM symbol $s_{n}$ can be computed in a similar way.

Equation (8) is similar to the MAP algorithm for nonbinary trellises derived in [10]. In the following, we derive this algorithm operating in the log domain. Although it is a well-known solution to avoid numerical instability and reduce the decoding complexity in iterative decoding, the TCM Log-MAP decoding is not well documented and described in the existing literature. Especially, the algorithm for the TCM decoding with channel value (in addition to symbol a priori information) is lacking. Therefore, we feel necessary to provide a detailed derivation of our TCM decoding scheme. Taking the logarithm of (8), we obtain

$$
\begin{aligned}
& L\left(s_{n}=s_{m} \mid \mathbf{y}\right)=\log \left(\sum_{\sigma^{\prime}, \sigma: s_{m}} e^{\bar{\alpha}_{n}\left(\sigma^{\prime}\right)+\bar{\gamma}_{n}^{*}\left(\sigma^{\prime}, \sigma\right)+\bar{\beta}_{n+1}(\sigma)}\right) \\
& \quad-\log \left(\sum_{\sigma^{\prime}, \sigma} e^{\bar{\alpha}_{n}\left(\sigma^{\prime}\right)+\bar{\gamma}_{n}\left(\sigma^{\prime}, \sigma\right)+\bar{\beta}_{n+1}(\sigma)}\right) \\
& =\max _{\sigma^{\prime}, \sigma: s_{m}} *\left\{\bar{\alpha}_{n}\left(\sigma^{\prime}\right)+\bar{\gamma}_{n}^{*}\left(\sigma^{\prime}, \sigma\right)+\bar{\beta}_{n+1}(\sigma)\right\} \\
& \quad-\max _{\sigma^{\prime}, \sigma}\left\{\bar{\alpha}_{n}\left(\sigma^{\prime}\right)+\bar{\gamma}_{n}\left(\sigma^{\prime}, \sigma\right)+\bar{\beta}_{n+1}(\sigma)\right\}
\end{aligned}
$$

We can also form the Log-MAP version of (9) as

$$
\begin{aligned}
& \lambda\left(b_{n}^{0}\right)= \log \frac{\sum_{\mathcal{B}^{+}} \exp \left[\bar{\alpha}_{n}\left(\sigma^{\prime}\right)+\bar{\gamma}_{n}\left(\sigma^{\prime}, \sigma\right)+\bar{\beta}_{n+1}(\sigma)\right]}{\sum_{\mathcal{B}^{-}} \exp \left[\bar{\alpha}_{n}\left(\sigma^{\prime}\right)+\bar{\gamma}_{n}\left(\sigma^{\prime}, \sigma\right)+\bar{\beta}_{n+1}(\sigma)\right]} \\
&=\max _{\mathcal{B}^{+}}\left[\bar{\alpha}_{n}\left(\sigma^{\prime}\right)+\bar{\gamma}_{n}\left(\sigma^{\prime}, \sigma\right)+\bar{\beta}_{n+1}(\sigma)\right] \\
& \quad-\max _{\mathcal{B}^{-}}\left[\bar{\alpha}_{n}\left(\sigma^{\prime}\right)+\bar{\gamma}_{n}\left(\sigma^{\prime}, \sigma\right)+\bar{\beta}_{n+1}(\sigma)\right]
\end{aligned}
$$

The notations used in (10) and (11) are defined as follows:

$$
\begin{aligned}
& L\left(s_{n}=s_{m} \mid \mathbf{y}\right)=\log P\left(s_{n}=s_{m} \mid \mathbf{y}\right) \\
& \bar{\alpha}_{n}\left(\sigma^{\prime}\right)=\log \alpha_{n}\left(\sigma^{\prime}\right), \quad \bar{\beta}_{n+1}(\sigma)=\log \beta_{n+1}(\sigma) \\
& \bar{\gamma}_{n}\left(\sigma^{\prime}, \sigma\right)=\log \gamma_{n}\left(\sigma^{\prime}, \sigma\right), \quad \bar{\gamma}_{n}^{*}\left(\sigma^{\prime}, \sigma\right)=\log \gamma_{n}^{*}\left(\sigma^{\prime}, \sigma\right)
\end{aligned}
$$

The branch metrics (probabilities) between states $\sigma^{\prime}$ and $\sigma$ are defined as

$$
\begin{aligned}
& \gamma_{n}\left(\sigma^{\prime}, \sigma\right)=p\left(y_{n}, \sigma \mid \sigma^{\prime}\right)=p\left(y_{n} \mid s_{n}\right) P\left(s_{n}\right) \\
& \gamma_{n}^{*}\left(\sigma^{\prime}, \sigma\right)=\gamma_{n}\left(\sigma^{\prime}, \sigma\right) / P\left(s_{n}\right)=p\left(y_{n} \mid s_{n}\right)
\end{aligned}
$$

Note that the a priori probability $P\left(s_{n}\right)$ is dropped from $\gamma_{n}^{*}\left(\sigma^{\prime}, \sigma\right)$ to avoid statistic dependencies between the soft values of several iteration steps, so that only the extrinsic probabilities are fed back to the equalizer.

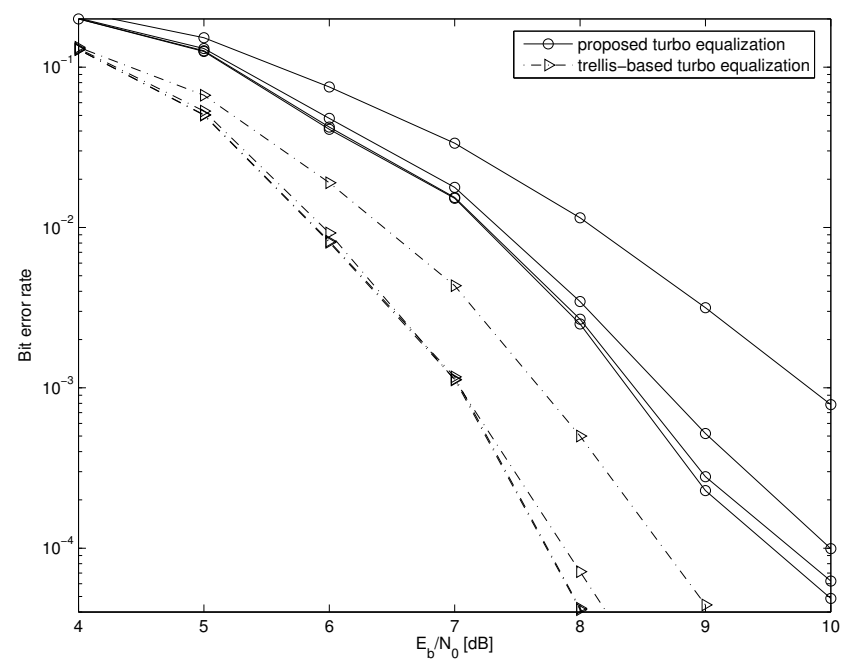

Fig. 3. Performance of filter and trellis-based turbo equalization for the 3-tap channel. Topmost curve represents the first iteration and the bottommost curve represents the $4^{\text {th }}$ iteration.

Assume accurate channel estimation, i.e., $\hat{\mathbf{h}} \approx \mathbf{h}$, then

$$
\begin{aligned}
& y_{n}=\hat{\mathbf{h}}^{*} \mathbf{h} s_{n}+\eta \approx \mathcal{P} s_{n}+\eta \\
& p\left(y_{n} \mid s_{n}=s_{m}\right)=\frac{1}{\pi N_{\eta}} \exp \left(-\frac{\left|y_{n}-\mathcal{P} s_{m}\right|^{2}}{N_{\eta}}\right) \\
& =\frac{1}{\pi \mathcal{P} N_{0}} \exp \left(-\frac{\left|y_{n}\right|^{2}+\mathcal{P}^{2}\left|s_{m}\right|^{2}-2 \mathcal{P} \operatorname{Re}\left\{s_{m}^{*} y_{n}\right\}}{\mathcal{P} N_{0}}\right) \\
& \propto \exp \left(\frac{2 \operatorname{Re}\left\{s_{m}^{*} y_{n}\right\}}{N_{0}}\right)
\end{aligned}
$$

where the common factor $\frac{1}{\pi \mathcal{P} N_{0}} \exp \left(-\frac{\left|y_{n}\right|^{2}+\mathcal{P}^{2}\left|s_{m}\right|^{2}}{\mathcal{P} N_{0}}\right)$ is omitted since it will be cancelled in the subsequent calculations. The branch metrics can be easily obtained by substituting (13) into (12).

\section{Numerical Results}

In our experiments, we employ Ungerboeck's 8-state $2 / 3$ 8PSK TCM. During each Monte-Carlo run, 4096 coded symbols are interleaved by an $64 \times 64$ block interleaver and transmitted over an ISI channel. For the initial equalization, we use a DFE equalizer with 7 feedforward, 3 feedback taps. It uses 200 pilot symbols for training the equalizer coefficients (using RLS). In the meantime, the maximum likelihood algorithm presented in [11] is used for channel estimation during training period. The channel coefficients are averaged over many estimated samples to reduce the noise effect.

Figure 3 shows the performance comparison between the trellis-based and the proposed scheme. Since the complexity of the trellis-based scheme increases exponentially with the number of channel taps, we choose a channel with very short impulse response: $h[n]=(\sqrt{0.3}+j \sqrt{0.3}) \delta[n]+(\sqrt{0.15}+$ $j \sqrt{0.15}) \delta[n-1]+(\sqrt{0.05}+j \sqrt{0.05}) \delta[n-2]$. To facilitate trellis-based equalization, two $s_{0}$ symbols are inserted to both the beginning and the end of the transmitted symbol sequence after interleaving to ensure the trellis decoding 
starts and ends at the zero state. As indicated in the figure, it takes 3 or 4 stages to reach convergence in both cases. Clearly, it is tremendous improvement by applying iterative process if we compare the topmost curve representing the separate equalization and decoding without feedback and the bottommost curve representing the turbo equalization and decoding after convergence. The trellis-based turbo equalization further improves the results at the cost of much higher computational complexity. The gap is between 1.5 to $1.8 \mathrm{~dB}$ for BER below $10^{-3}$. The performance loss by applying filter-based approach is owing to the fact that the symbol detection is made without taking into account the structure of the ISI channel, i.e., symbols are assumed to be independent. Assuming the elements of the input sequence come from $M$-ary alphabet, and the ISI channel has $L$ taps, the complexity of the trellis-based approach is $O\left(M^{L-1}\right)$. For channels with long memory and large constellation size, it becomes prohibitively complex. While the complexity of the proposed scheme is only $O\left(L^{2}\right)$, which makes it feasible for practical implementation.

The performance of the proposed scheme and the MMSE filter-based turbo equalization scheme is compared in Figure 3, where we use a 5-tap channel with impulse response $h[n]=(2-0.4 j) \delta[n]+(1.5+1.8 j) \delta[n-1]+\delta[n-2]+(1.2-$ $1.3 j) \delta[n-3]+(0.8+1.6 j) \delta[n-4]$. The output channel power is normalized so that $P=\sum_{n=0}^{4}|h[n]|^{2}=1$. As shown in the figure, the proposed scheme outperforms the original MMSE filer-based turbo equalization by $0.6 \mathrm{~dB}$ around $\mathrm{BER}=10^{-4}$. In addition to the original scheme introduced in $[6,7]$, we also tested its improved version and pass the MMSE filter output to the TCM decoder in addition to the symbol a posteriori probabilities. Clearly, the improved algorithm performs better than the original one. This means that it is necessary for the TCM decoder to take the refined channel value as input in order to improve the decoding performance. The plot also shows that the proposed algorithm yields identical performance at low SNR and slightly superior performance at high SNR to the improved version of the MMSE filter-based turbo equalization. While the former reduces the complexity from $O\left(L^{3}\right)$ to $O\left(L^{2}\right)$ by avoiding the MMSE filtering and matrix inversion in the equalization process.

\section{Conclusions}

Filter-based turbo equalization overcomes the prohibitive complexity imposed by trellis-based approach, and is shown to be a feasible solution for systems with high spectral efficiency modulation schemes and channels exhibiting a large delay spread. In this paper, a novel approach to joint turbo equalization and TCM decoding scheme is investigated. Unlike the existing filter-based equalization scheme, the proposed algorithm does not involve MMSE filtering and matrix inversion, it reduces the complexity from cubic to quadratic. Furthermore, by passing the refined channel value from the equalizer to the TCM decoder, the quality of decoding is also improved. Simulation results of an 8-PSK TCM system show that the proposed scheme outperforms

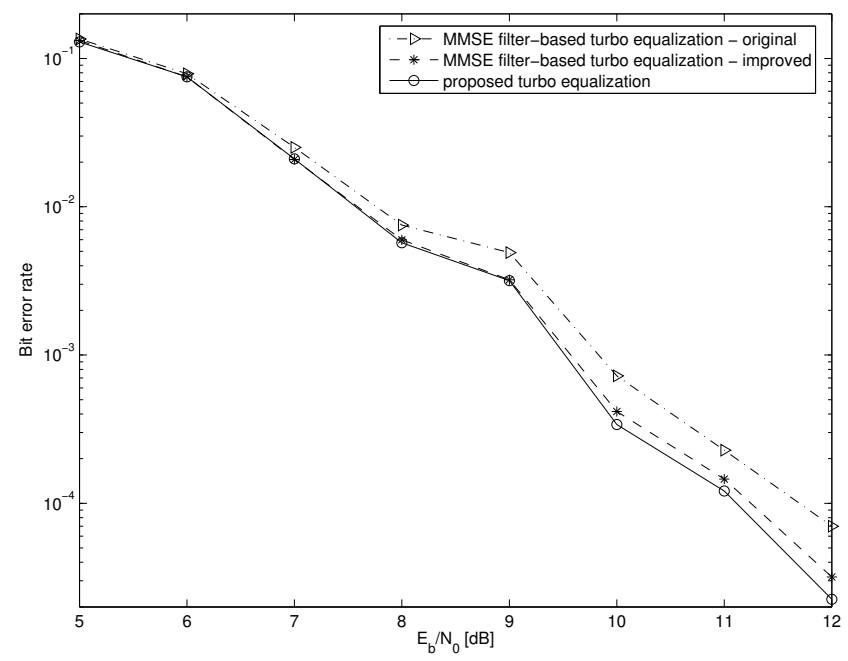

Fig. 4. Comparison between the proposed and the MMSE filter-based turbo equalization for the 5-tap channel. All the curves represent the $4^{t h}$ stage turbo equalization.

the existing MMSE filter-based turbo equalization with reduced complexity.

\section{REFERENCES}

[1] C. Douillard. "Iterative correction of intersymbol interference: turbo-equalization". European Transactions on Telecommunications, pp. 507-511, Sept. 1995.

[2] X. Wang, H. Poor. "Iterative (turbo) soft interference cancellation and decoding for coded CDMA". IEEE Transactions on Communications, vol. 47, no. 7, pp. 1046-1061, July 1999.

[3] M. Tuchler, R. Koetter, A. Singer. "Turbo equalization: principles and new results". IEEE Transactions on Communications, vol. 50, pp. 754-767, May 2002.

[4] N. Doan, R. Rajatheva. "Turbo equalization for non-binary coded modulation schemes over frequency selective fading channels". In Proc. IEEE Vehicular Technology Conference, vol. 3, pp. 2217 2221, May 2000.

[5] P. Magniez, P. Duhamel. "Turbo-equalization applied to trelliscoded-modulation". In Proc. IEEE Vehicular Technology Conference, pp. 2556-2560, 1999.

[6] A. Dejonghe, L. Vandendorpe. "Turbo-equalization for multilevel modulations: an efficient low-complexity scheme". In Proc. IEEE International Conference on Communications, vol. 3, pp. 18631867, April - May 2002.

[7] M. Koca, B. Levy. "Broadband beamforming for joint interference cancellation and low-complexity turbo equalization". In Proc. IEEE Global Telecommunications Conference, vol. 4, pp. 2161-2166, Dec. 2003.

[8] G. Ungerboeck. "Channel coding with multilevel/phase signals". IEEE Transactions on Information Theory, vol. 28, pp. 56-67, Jan. 1982.

[9] L. Bahl, J. Cocke, F. Jelinek, J. Raviv. "Optimal decoding of linear codes for minimizing symbol error rate". IEEE Transactions on Information Theory, vol. 20, pp. 284-287, 1974.

[10] P. Robertson, T. Worz. "Bandwidth-efficient turbo trellis-coded modulation using punctured component codes". IEEE Selected Areas in Communications, vol. 16, no. 2, Feb 1998.

[11] E. Ström, S. Miller. "Iterative demodulation and channel estimation of orthogonal signalling formats in asynchronous DS-CDMA systems". IEICE Transactions on Electronics, vol. E85-C, no. 3, pp. 442-451, March, 2002. 